\title{
Design of Non-Uniform Antenna Arrays Using Genetic Algorithm
}

\author{
Murad Ridwan ${ }^{1, *}$, Mohammed Abdo ${ }^{1}$, Eduard Jorswieck ${ }^{2}$ \\ ${ }^{1}$ Department of Electrical \& Computer Engineering, Addis Ababa University, Ethiopia \\ ${ }^{2}$ Communications Laboratory, Dresden University of Technology \\ muradridwan@yahoo.com,abdo@ece.aau.edu.et, jorswieck@ifn.et.tu-dresden.de
}

\begin{abstract}
The performance of a single-element antenna is somewhat limited. To obtain high directivity, narrow beamwidth, low side-lobes, point-to-point and preferred-coverage pattern characteristics, etc., antenna arrays are used. An antenna array is an assembly of individual radiating antennas in an electrical and geometrical configuration. Nowadays, antenna arrays appear in wireless terminals and smart antennas, so robust and efficient array design is increasingly becoming necessary. In antenna array design, it is frequently desirable to achieve both a narrow beamwidth and a low side-lobe level. In linear antenna arrays, a uniform array yields the smallest beamwidth and hence the highest directivity. It is followed, in order, by the Dolph-Chebyshev and Binomial arrays. In contrast, Binomial arrays usually possess the smallest side-lobes followed, in order, by the Dolph-Chebyshev and uniform arrays.Binomial and Dolph-Chebyshev arrays are typical examples of non-uniform arrays. In this paper we deal only with linear arrays and it is shown that using genetic algorithm it is possible to design a non-uniform array that approximates the beamwidth of a uniform array and having smaller side-lobe level than the Dolph-Chebyshev array. The result is that the designed antenna array exhibits the largest directivity as compared to the uniform, Binomial and Dolph-Chebyshev arrays. In the design, the genetic algorithm is employed to generate the excitation amplitudes of the antenna array.
\end{abstract}

Keywords Antenna Arrays, Uniform Arrays, Non-Uniform Arrays, Binomial Arrays, Dolph-Chebyshev Arrays, Genetic Algorithm

\section{Introduction}

The performance of a single-element antenna is somewhat limited. To obtain high directivity, narrow beamwidth, low side-lobes, point-to-point and preferred-coverage pattern characteristics, etc., antenna arrays are used[1]. An antenna array, or simply array, is an assembly of individual radiating antennas in an electrical and geometrical configuration.

The design of an array involves mainly first the selection of elements and array geometry, and then the determination of the element excitations required for achieving a particular performance.

Over the past decades, the theory of antenna array design has been studied in depth and is certainly well documented[1]. But recently the applications of Genetic Algorithms (GAs) to engineering electromagnetics[2,4] and antenna array design and synthesis[5,9] have attracted much attention.

This paper is organized as follows. The system model is presented in Section 2. Section 3 describes the genetic

* Corresponding author:

muradridwan@yahoo.com (Murad Ridwan)

Published online at http://journal.sapub.org/jwnc

Copyright (C 2012 Scientific \& Academic Publishing. All Rights Reserved algorithm as applied in the context of non-uniform arrays. In Section 4, we provide some representative numerical results. Finally in Section 5 concluding remarks are made.

\section{Problem Formulation}

Consider a uniformly spaced linear array of $N$ elements places along the $z$-axis. Supposethe distance between any two adjacent elements is $d$ and the array is operated at $\lambda$. The array factor is

$$
A F(\theta)=\sum_{n=1}^{N} a_{n} e^{j(2 \pi n d \cos \theta) / \lambda}
$$

The feeding coefficient $a_{n}$ can be written as

$$
a_{n}=\left|a_{n}\right| e^{j \alpha_{n}}(2)
$$

In uniform arrays, the elements have equal amplitude and a constant phase difference between adjacent ones, i.e.,

$$
a_{n}=a_{o} e^{-j n \beta}(3)
$$

where $\beta$ is the progressive phase shift.

In binomial and Dolph-Chebyshev arrays, which are typical examples of non-uniform arrays, the amplitude excitations are derived from the binomial series and Chebyshev's polynomials, respectively[1].

As stated in the abstract, in antenna system design it is frequently desirable to achieve both a narrow beamwidth and 
a low side-lobe level[10]. A uniform array yields the smallest beamwidth and the highest directivity. It is followed, in order, by the Dolph-Chebyshev and binomial arrays. In contrast, binomial arrays usually possess the smallest side-lobes followed by, in order, by the Dolph-Chebyshev and uniform arrays[1].

The objective of the present paper is to design, using GA, a non-uniform linear array that approximates the beamwidth of uniform arrays and produces smaller side-lobe level than that of Dolph-Chebyshev arrays. The array so designed willposses, as will be shown, the highest directivity than uniform, binomial, and Dolph-Chebyshev arrays.

From Equation (1), the array factor for a non-uniform broadside $\left(\alpha_{n}=0\right)$ array is

$$
A F(\theta)=\sum_{n=1}^{N} a_{n} e^{j(2 \pi n d \cos \theta) / \lambda}
$$

where, for simplicity, we let $a_{n}=\left|a_{n}\right|$ since $\alpha_{n}=0$ (broadside).

To design the array, first the number of array elements $N$ and the inter-element spacing $d$ are specified. The genetic algorithm generates $N$ excitation coefficients $a_{n}$ and forms the non-uniform array factor $A F(\theta)$ (Equation 4). By successive generation of $a_{n}$ 's through the GA, the desired array factor is made to approximate a hypothetical array factor $A F_{h}(\theta)$ in some acceptable sense. The array factor $A F_{h}(\theta)$ is one which, in the region of the main lobe, is the same as a uniform broadside array with the same $N$ and $d$, but is zero elsewhere-i.e., with no side-lobes.

$$
A F_{h}(\theta)=\left\{\begin{array}{c}
\frac{1}{N} \frac{\sin \left(\frac{\pi N d}{\lambda} \cos \theta\right)}{\sin \left(\frac{\pi d}{\lambda} \cos \theta\right)}, \text { for } \cos ^{-1}\left(\frac{\lambda}{N d}\right) \leq \theta . . \\
. . \leq \frac{\pi}{2}+\cos ^{-1}\left(\frac{\lambda}{N d}\right) \\
0, \text { otherwise }
\end{array}\right.
$$

Since the required non-uniform array $A F(\theta)$ is to approximate $A F_{h}(\theta)$, the mean-squared error of the difference between $A F(\theta)$ and $A F_{h}(\theta)$ is passed to the genetic algorithm as an objective or fitness function, $f\left(\theta ; a_{1}, \cdots, a_{N}\right)$, to be minimized, where

$$
\begin{aligned}
& f\left(\theta ; a_{1}, \cdots, a_{N}\right)=\left\|A F(\theta)-A F_{h}(\theta)\right\|^{2} \text {, or } \\
& f=\left\{\begin{array}{l}
\left\|\sum_{n=1}^{N} a_{n} e^{j(2 \pi n d \cos \theta) / \lambda}-\frac{1}{N} \frac{\sin \left(\frac{\pi N d}{\lambda} \cos \theta\right)}{\sin \left(\frac{\pi d}{\lambda} \cos \theta\right)}\right\|^{2} \\
\text { for } \cos ^{-1}\left(\frac{\lambda}{N d}\right) \leq \theta \leq \frac{\pi}{2}+\cos ^{-1}\left(\frac{\lambda}{N d}\right)(6) \\
\left\|\sum_{n=1}^{N} a_{n} e^{j(2 \pi n d \cos \theta) / \lambda}\right\|^{2}, \quad \text { otherwise }
\end{array}\right.
\end{aligned}
$$

\section{The GA as Applied to Non-UniformLinear Antenna Array Design}

The Genetic Algorithm is a method for solving optimization problems that is based on the evolutionists' natural selection concept. The GA repeatedly modifies a population of individual solutions. At each step, the GA selects individuals at random from the current population to be parents and uses them produce the children for the next generation. Over successive generations, the population "evolves" toward an optimal solution. At each step of the iteration, the GA uses the selection, crossover and mutation rules to create the next generation from the current population.

The GA can be applied to solve a variety of optimization problems that are not well suited for standard optimization algorithms, including problems in which the objective function is discontinuous, non-differentiable, stochastic, or highly nonlinear.

The algorithm begins by creating a random initial population- in our case, a set of random excitation amplitudes in a given range, say, twenty sets of $N a_{n}$ 's. The algorithm then creates a sequence of new populations, or generations. At each step, the algorithm uses the individuals in the current generation to create the next generation. To create the new generation, the algorithm performs the following steps: scores each member of the current population by computing its fitness value. The fitness value of an individual is the value of the fitness function for that individual. The fitness or objective function is the function to be optimized. In the non-uniform array context, the fitness function is the mean-squared error of the difference between the array factors generated by individual members of a generation and the hypothetical side-lobe free uniform array (Equation 6). The algorithm then selects parents with the lowest fitness values. Children are produced from parents either by making random changes to a single parent- mutation, or by combining the vector entries of a pair of parents- crossover. The current population is replaces with the children to form the next generation. Finally the algorithm stops when one of the stopping criteria is met. Usually the stopping criteria is when the number of generations reaches a pre-set maximum number of generations or when the value of the fitness function for the best point in the current population is less than or equal to a pre-set fitness value.

\section{Results}

As it is well known in optimization problems, the number of local minima gets on exploding as the number of optimization variables increase. This is to be expected in antenna array design, since the number of nulls increase as $N$ increases. Compared to standard optimization algorithms, the genetic algorithm is strong at escaping the local minima[12].

The non-uniform array designed using the genetic algorithm exhibits the highest directivity, will have a narrow beamwidth comparable to uniform array and displays lower side-lobe levels than Dolph-Chebyshev or uniform arrays. In Dolph-Chebyshev arrays, the side-lobe levels are assumed to be $20 \mathrm{~dB}$ below the maximum of the major lobe.Some rep- 
resentative examples will be considered.

Example1: For a linear array with $N=10$ and $d=0.25 \lambda$, a broadside uniform, binomial, and Dolph-Chebyshev arrays are produced. With the same $N$ and $d$, a non-uniform array is designed using the genetic algorithm. The result is displayed in Figure 1. The excitation coefficients of the non-uniform array as produced by the genetic algorithm are:

$$
\begin{array}{ll}
a_{1}=1.5051 & a_{2}=0.8778 \\
a_{3}=-0.4210 & a_{4}=3.5444 \\
a_{5}=-0.3286 & a_{6}=0.9350 \\
a_{7}=2.3436 & a_{8}=0.5822 \\
a_{9}=0.2470 & a_{10}=1.7174
\end{array}
$$

The directivities in descending order are shown in Table I.

Table 1.Directivities for Example 1

\begin{tabular}{|c|c|}
\hline Array & Directivity \\
\hline Using GA & 5.4382 \\
\hline Uniform & 5.1658 \\
\hline Dolph-Chebyshev & 4.9253 \\
\hline Binomial & 2.6967 \\
\hline
\end{tabular}

As claimed earlier, the array designed using the genetic algorithm exhibits the largest directivity while keeping its beamwidth as narrow as the uniform array.

Example 2: As in Example 1 above, but with $N=20$ and $d$ $=0.25 \lambda$, the result is displayed in Figure 2. The excitation coefficients are:

$$
\begin{array}{ll}
a_{1}=1.4647 & a_{2}=-0.0557 \\
a_{3}=0.7709 & a_{4}=1.6612 \\
a_{5}=0.2775 & a_{6}=1.1205 \\
a_{7}=0.9641 & a_{8}=1.6770 \\
a_{9}=0.6786 & a_{10}=0.2991 \\
a_{11}=3.1648 & a_{12}=-0.5235 \\
a_{13}=1.3070 & a_{14}=1.6972 \\
a_{15}=1.0998 & a_{16}=-0.1354 \\
a_{17}=1.7464 & a_{18}=1.0624 \\
a_{19}=-0.4338 & a_{20}=1.4978
\end{array}
$$

The directivities in descending order are:

Table 2.Directivities for Example 2

\begin{tabular}{|c|c|}
\hline Array & Directivity \\
\hline Using GA & 10.1630 \\
\hline Uniform & 10.1593 \\
\hline Dolph-Chebyshev & 10.0092 \\
\hline Binomial & 3.8884 \\
\hline
\end{tabular}

Example 3: When the inter-element spacing $d$ becomes larger, the number of side-lobes proportionally increases. This means the fluctuations between nulls and side-lobe peaks increases and the efficiency of the genetic algorithm decreases. This effect can be illustrated by taking $N=10$ and $d=0.5 \lambda$. The result is displayed in Figure 3 and the excitation coefficients are:

$$
\begin{array}{ll}
a_{1}=0.4950 & a_{2}=0.6905 \\
a_{3}=0.7415 & a_{4}=0.8094 \\
a_{5}=0.8379 & a_{6}=0.8401 \\
a_{7}=0.8126 & a_{8}=0.7458 \\
a_{9}=0.6782 & a_{10}=0.5018
\end{array}
$$

The directivities in descending order are:

Table 3.Directivities for Example 3

\begin{tabular}{|c|c|}
\hline Array & Directivity \\
\hline Uniform & 9.9980 \\
\hline Using GA & 9.7197 \\
\hline Dolph-Chebyshev & 9.6203 \\
\hline Binomial & 5.3914 \\
\hline
\end{tabular}

Example 4: If the inter-element spacing $d$ becomes smaller, the number of side-lobes proportionally decreases. The array factor becomes smoother. This increases the efficiency of the genetic algorithm. The effect can be illustrated by taking $N=$ 10 and $d=0.15 \lambda$. The result is displayed in Figure 4 . The excitation coefficients are:

$$
\begin{array}{ll}
a_{1}=-2.8430 & a_{2}=-1.0364 \\
a_{3}=-0.5084 & a_{4}=-0.0046 \\
a_{5}=-3.3524 & a_{6}=-1.5005 \\
a_{7}=-3.2832 & a_{8}=1.2169 \\
a_{9}=-0.0279 & a_{10}=-4.1288
\end{array}
$$

The directivities in descending order are:

Table 4.Directivities for Example 4

\begin{tabular}{|c|c|}
\hline Array & Directivity \\
\hline Using GA & 3.6364 \\
\hline Uniform & 3.2045 \\
\hline Dolph-Chebyshev & 3.2045 \\
\hline Binomial & 1.6826 \\
\hline
\end{tabular}

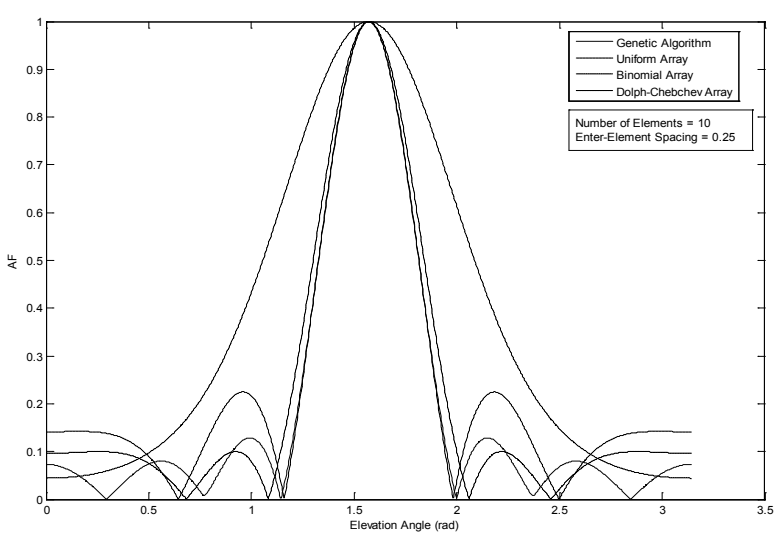

Figure 1. Magnitude of normalized array factor, $|\mathrm{AF}|$, versus $\theta$ for $N=10$, $d=0.25 \lambda$

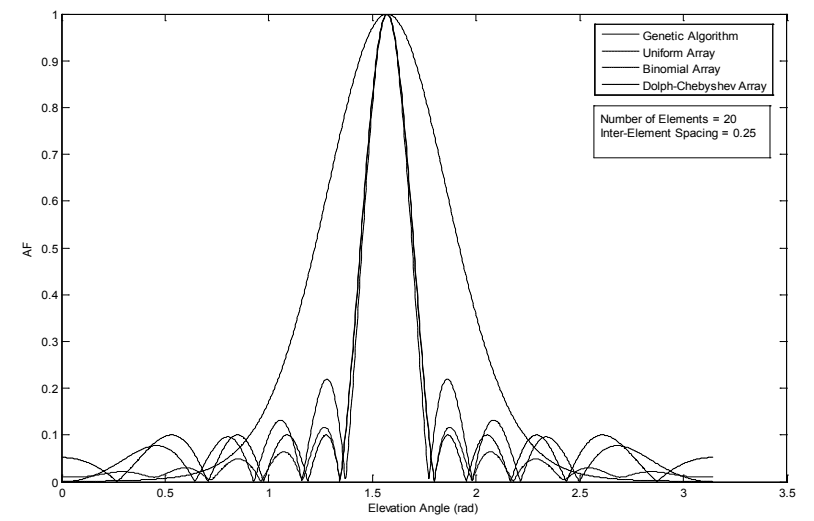

Figure 2. Magnitude of normalized array factor, $|\mathrm{AF}|$, versus $\theta$ for $N=20$, $d=0.25 \lambda$ 


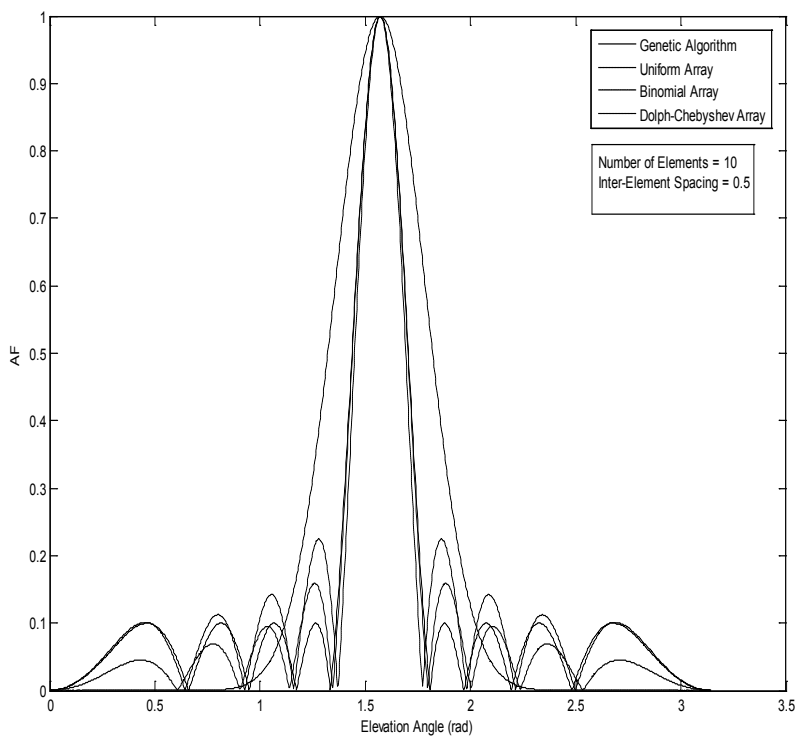

Figure 3. Magnitude of normalized array factor, $|\mathrm{AF}|$, versus $\theta$ for $N=10$, $d=0.5 \lambda$

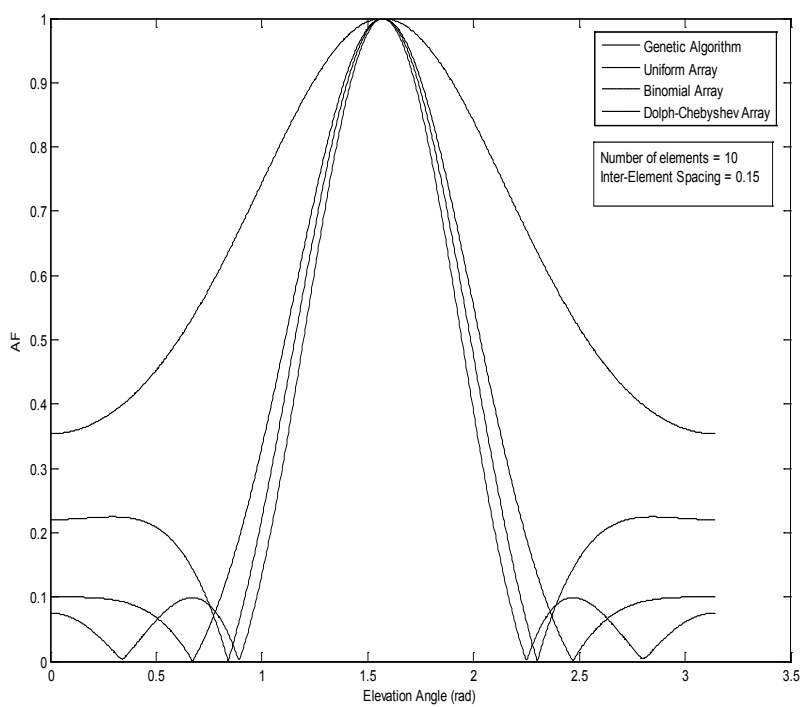

Figure 4. Magnitude of normalized array factor, $|\mathrm{AF}|$, versus $\theta$ for $N=10$, $d=0.15 \lambda 5$. Conclusions

\section{Conclusions}

As demonstrated above, genetic algorithm can be used to design an efficient non-uniform array. The designed array exhibits the largest directivity and a beamwidth approximating that of uniform arrays. Besides, the side-lobe level is smaller than that of either uniform or Dolph-Chebyshev arrays.

For inter-element spacing greater than $0.5 \lambda$, the efficiency of the designed array relatively decreases as opposed to smaller spacing. This is not a problem in practical terms since real arrays will have inter-element spacing less than or equal to $0.5 \lambda$ to avoid large number of side-lobes and perhaps grating lobes.

\section{REFERENCES}

[1] C. A. Balanis, Antenna Theory: Analysis and Design, 3rd ed. New York: Wiley, 2005.

[2] Y. Rahmat-Samii and E. Michielssen, eds., Electromagnetic Optimization by Genetic Algorithms. New York: Wiley, 1999.

[3] J. M. Johnson and Y. Rahmat-Samii, "Genetic Algorithms in Engineering Electromagnetic,"IEEE Antennas Propag. Mag., vol. 39, no. 4, pp. 7-25, 1997.

[4] D. E. Goldberg, Genetic Algorithms in Search, Optimization and Machine Learning. Reading, MA: Addison-Wesley, 1989.

[5] D. W. Boeringer, D. H.Werner, and D. W. Machuga, "A simultaneous parameter adaptation scheme for genetic algorithms with application to phased array synthesis," IEEE Trans. Antennas Propag., vol. 53, pp. 356-371, Jan. 2005.

[6] Y. B. Tian and J. Qian, "Improve the performance of a linear array by changing the spaces among array elements in terms of genetic algorithm," IEEE Trans. Antennas Propag., vol. 53, pp. 2226-2230, Jul. 2005.

[7] W. P. Liao and F. L. Chu, "Array pattern synthesis with null steering using genetic algorithm by controlling only the current amplitudes," Int. J. Elect., vol. 86, no. 4, pp. 445-457, 1999.

[8] B. K. Yeo and Y. Lu, "Array failure correction with a genetic algorithm," IEEE Trans. Antennas Propag., vol. 47, no. 5, pp. 823-828, May 1999.

[9] K. K. Yan and Y. Lu, "Side-lobe reduction in array-pattern synthesis using genetic algorithm," IEEE Trans. Antennas Propag., vol. 45, no. 7, pp. 1117-1121, Jul. 1997

[10] W.L. Weeks, Antenna Engineering, McGraw-Hill, New York, 1968.

[11] R. Jain, and G. S. Mani, "Dynamic thinningofantenna array using genetic algorithm", Progress In Electromagnetics Re$\operatorname{searchB}$, Vol. 32, 1-20, 2011.

[12] Francisco J. Ares-Pena, Juan A. Rodriguez-Gonzalez, E. Villanueva-Lopez, and S. R. Rengarajan, "Genetic Algorithms in the Design andOptimization of Antenna Array Patterns", IEEE Trans. Antennas Propagat., vol. 47, no. 3, March 1999. 